\section{Enfermedad coronaria en mujeres pre y postmenopáusicas y su relación con la diabetes mellitus tipo 2}

\author{
JORGE GAJARDO-NAVARRETE ${ }^{1}$, GUILLERMO IBIETA ${ }^{1}$, \\ MARCO CONCHA ${ }^{1}$, PAMELA GARCÉS ${ }^{1}$, ISABEL ROBLES ${ }^{3, c}$, \\ ALDO VERA-CALZARETTA ${ }^{2, \mathrm{a}, \mathrm{d}}$, ALEXIS GONZÁLEZ-BURBOA ${ }^{2, \mathrm{~b}, \mathrm{e}}$, \\ IGNACIO GAJARDO ${ }^{4, \mathrm{f}}$, MILENKA GUTIÉRREZ ${ }^{4, \mathrm{f}}$, \\ MAURICIO VILLARROEL ${ }^{4, f}$, MATÍAS AGUAYO ${ }^{4, f}$
}

\section{Coronary artery disease in pre and postmenopausal women. The influence of type 2 diabetes mellitus}

Background: Postmenopausal women have higher severity of coronary heart disease (CHD) than premenopausal women and type 2 diabetes mellitus (T2DM) is an independent risk factor. Aim: To assess the severity of CHD in pre and postmenopausal patients undergoing coronary angiography and the impact of T2DM in both groups. Material and Methods: A coronary angiography was performed to 707 women due to suspected CHD during 2013 and 2014. Of these, 579 were older than 55 years and were considered as postmenopausal. Factors such as hypertension, obesity, smoking, creatinine and T2DM were registered. The severity of CHD in coronary angiography was evaluated according to the number of vessels with more than 50\% stenosis. Results: Compared to their postmenopausal counterparts, premenopausal women had less frequency of T2DM (31\% and 42\% $p<0.033)$, hypertension (52 and 78\%, $p<0.001$ ) and alteration of renal function (11 vs. 39\%, $p<0.001$ ). Absence of coronary lesions was found in 44 and 32\% of premenopausal and postmenopausal women, respectively $(p<0.01)$. Premenopausal women with T2DM had a higher frequency of multi-vessel disease than those without the disease (25 and 4.5\%, $p<0.001)$. The frequency of multi-vessel disease was higher in postmenopausal than premenopausal women (24 and 11\%, $p<0.01$ ). Hypertension, T2DM and renal involvement were associated with a higher frequency multiple vessel disease. Conclusions: The severity of CHD is higher in postmenopausal women and T2DM is associated with the disease.

(Rev Med Chile 2020; 148: 145-150)

Key words: Coronary Disease; Diabetes Mellitus; Postmenopause; Women.

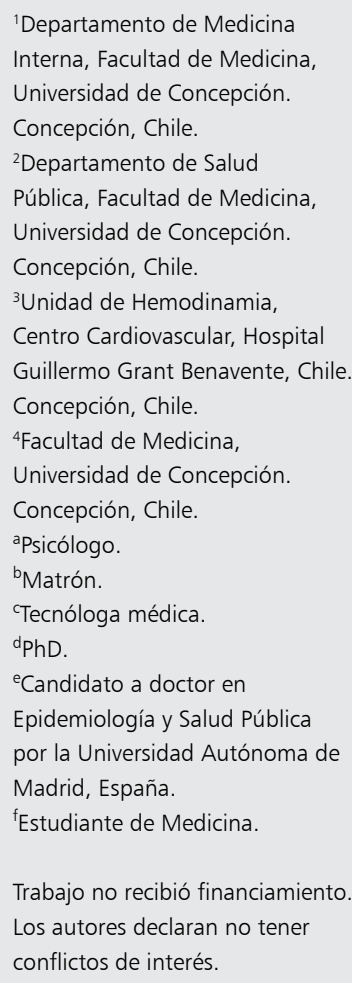

Recibido el 22 de agosto de 2019, aceptado el 6 de febrero de 2020.

Correspondencia a: Jorge Gajardo Navarrete, MD. Departamento de Especialidades Médicas. Facultad de Medicina. Universidad de Concepción. Avda. Chacabuco esquina Janequeo $s / n, 4^{\circ}$ piso. Concepción, Chile. jgncardio@gmail.com

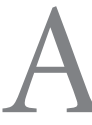

un cuando la frecuencia de la enfermedad coronaria (EC) aumenta con la edad en ambos sexos, esto se ha ido modificando desde la década 1950-59 a la fecha con tendencia a declinar entre los hombres y a aumentar entre las mujeres ${ }^{1}$, en las cuales la EC se retarda aproxi- madamente una década respecto de los hombres ${ }^{2}$. Esto último está dado porque las mujeres jóvenes tienen el efecto protector de los estrógenos, lo cual ayuda a prevenir la aterosclerosis ${ }^{3}$. Dicho efecto se pierde en la postmenopausia, asociándose, además, a una mayor prevalencia de múltiples factores 
de riesgo como hipertensión arterial (HTA), obesidad y diabetes mellitus tipo 2 (DM2), los cuales, generalmente, van aumentando su prevalencia a medida que las personas aumentan en edad. En el año 1994, el National Cholesterol Education Program (NCEP $)^{4}$ reconoció a la postmenopausia como un factor de riesgo cardiovascular.

La enfermedad coronaria es una de las primeras causas de mortalidad en mujeres postmenopáusicas ${ }^{5,6}$ y se han reportado diferencias en síntomas, factores de riesgo, pronóstico y recurrencia de enfermedad coronaria entre mujeres pre y postmenopáusicas ${ }^{7,8}$. Algunas publicaciones ${ }^{8,9}$ han hecho referencia a un mayor compromiso de las arterias coronarias en las postmenopáusicas.

Poca información existe en nuestro país sobre el real impacto de la postmenopausia en la gravedad y extensión de la EC con o sin DM2 asociada.

Este trabajo tuvo como hipótesis que la mujer postmenopáusica presenta EC más extensa y más grave que la premenopáusica, y que la DM2 empeora aquello en ambos grupos de pacientes.

\section{Material y Método}

\section{Diseño del estudio}

Estudio de corte transversal, de carácter descriptivo.

\section{Sujetos}

Participaron del estudio 707 mujeres que fueron estudiadas con coronariografía en forma consecutiva entre el 1 de enero de 2013 y el 31 de diciembre de 2014 en el Laboratorio de Hemodinamia del Hospital Guillermo Grant Benavente (HGGB) de la ciudad de Concepción.

Para diferenciar a las mujeres premenopáusicas (grupo 1) de las postmenopáusicas (grupo 2), se utilizó como criterio de corte la edad de 55 años. De este modo, el grupo 1 quedó constituido por 128 mujeres menores de 55 años y el grupo 2 por 579 mujeres con edades iguales o mayores a 55 años.

El estudio fue indicado por diversas causas, a saber, la existencia de patologías valvulares, una respuesta positiva para insuficiencia coronaria frente un test de esfuerzo, la presencia de cuadros coronarios estables, o de síndromes coronarios agudos.

Se excluyeron aquellas pacientes con cirugía de revascularización previa, aneurisma aórtico, miocarditis, cardiomiopatías, tromboembolismo pulmonar y enfermedad cardiaca congénita.

Todas las mujeres fueron estudiadas con coronariografía según técnica estándar, bajo anestesia local, por vía radial izquierda o derecha.

\section{Levantamiento de la información}

La información utilizada en este estudio fue obtenida en forma retrospectiva y anónima desde los registros médicos y de enfermería del Laboratorio de Hemodinamia del HGGB de la ciudad de Concepción. La anonimización fue llevada a cabo por un profesional designado por la jefatura del servicio clínico, quien, a su vez proveyó la misma al responsable del estudio y tuvo por rol resguardar su cumplimiento. Los análistas de datos solo accedieron a los datos en la medida en que esto fue facilitado por el investigador responsable.

Desde los registros se obtuvo la edad, el diagnóstico cardiológico clínico, factores de riesgo tales como HTA, obesidad, tabaquismo, DM2 y la descripción de la coronariografía en el informe del laboratorio de hemodinamia. La función renal al momento del estudio también fue registrada.

La gravedad de la enfermedad coronaria fue evaluada según el número de vasos comprometidos $(0,1,2,3$ vasos, respectivamente) con enfermedad obstructiva mayor de $50 \%$ de reducción del diámetro luminal de la arteria en la coronariografía. Se consideró como múltiples vasos el compromiso de 2 o más arterias coronarias epicárdicas.

\section{Análisis estadísticos}

Se llevó a cabo un análisis exploratorio de los datos, calculando promedio y desviación estándar junto con frecuencias absolutas y relativas. Para la comparación de variables cuantitativas en dos muestras independientes se utilizó el test $t$ de Student. Por otra parte, en el estudio de la asociación de dos variables se utilizó la prueba de independencia de $\chi^{2}$ tratándose de variables nominales con dos o más categorías. Finalmente, se realizaron regresiones logísticas simples y múltiple para la variable respuesta compromiso de múltiples vasos (donde $1=$ múltiples vasos; $0=$ enfermedad mínima o sin enfermedad).

El nivel de significancia para este estudio se estableció con un valor $\mathrm{p}<$ de 0,05 .

Este estudio fue aprobado por el Comité Ético Científico del Servicio de Salud Concepción. 


\section{Resultados}

La edad promedio de las pacientes premenopáusicas y postmenopáusicas fue de 47,8 . $(\mathrm{DE}=5,1)$ y $69(\mathrm{DE}=8,4)$ años, respectivamente. Las mujeres premenopáusicas tenían menos DM2 $(31,3 \%$ vs $41,5 \%)(\mathrm{p}<0,033)$, menos hipertensión $(51,6 \%$ vs $77,9 \%)(\mathrm{p}<0,001)$ y menos compromiso de la función renal $(11,1 \%$ vs $38,6 \%)(\mathrm{p}<0,001)$ que las mujeres postmenopáusicas. La arteria más comúnmente comprometida fue la descendente anterior, seguida por la coronaria derecha en ambos grupos de pacientes (Tabla 1).

El 43,8\% de las premenopáusicas estaba libre de EC angiográfica vs 32\% de las postmenopáusicas $(\mathrm{p}<0,01)$. Las postmenopáusicas sin DM2 tenían más enfermedad de múltiples vasos que las premenopáusicas sin DM2 (17,1\% vs $4,5 \%)$
( $\mathrm{p}<0,001)$. Las premenopáusicas con DM2 tenían más enfermedad de múltiples vasos ( $25 \%$ vs $4,5 \%)(\mathrm{p}<0,001)$ y más oclusión crónica $(27,5 \%$ vs $1,1 \%)$ que las premenopáusicas sin DM2.

En el grupo de las mujeres postmenopáusicas, aquellas con DM2 tenían más enfermedad de múltiples vasos $(33,3 \%$ vs $17,1 \%)(\mathrm{p}<0,001)$ y más oclusión crónica $(21,8 \%$ vs $14,7 \%)(\mathrm{p}<0,001)$ que las sin DM2.

Como grupo total, las postmenopáusicas tenían significativamente más enfermedad de múltiples vasos $(23,8 \%$ vs $10,9 \%)$ y más arterias con oclusión crónica $(17,6 \%$ vs $9,4 \%)$ que las premenopáusicas $(\mathrm{p}<0,01)$ (Tabla 2$)$.

Al considerar como variable de ajuste la DM2, observamos que ambos grupos de pacientes con DM2 tenían mayor compromiso de múltiples vasos. En el análisis de regresión múltiple las va-

Tabla 1. Características sociodemográficas y antecedentes mórbidos de las participantes según pre y postmenopausia

\begin{tabular}{|c|c|c|c|}
\hline & Mujeres premenopaúsica & Mujeres postmenopáusica & Valor $\mathbf{p}^{*}$ \\
\hline \multicolumn{4}{|c|}{ Sociodemográficas, $\pm D E^{*}$} \\
\hline Edad (años) & $47,8 \pm 5,1$ & $69,5 \pm 8,4$ & 0,001 \\
\hline \multicolumn{4}{|c|}{ Antecedentes mórbidos, $\mathrm{n}(\%)^{*}$} \\
\hline Hipertensión & $66(51,6)$ & $451(77,9)$ & 0,001 \\
\hline Diabetes mellitus & $40(31,3)$ & $240(41,5)$ & 0,036 \\
\hline Tabaquismo & $36(28,8)$ & $75(13,2)$ & 0,001 \\
\hline $\mathrm{IMC}^{\dagger}$ & $37(40,0)$ & $170(34,5)$ & 0,528 \\
\hline VFG $<60^{*}$ & $11(11,1)$ & $187(38,6)$ & 0,001 \\
\hline
\end{tabular}

${ }^{*} p \leq 0,05 .{ }^{¥}$ Prueba t de Student para muestras independientes; ${ }^{\sharp}$ Prueba de independencia de $\chi^{2}$; IMC: índice de masa corporal VFG: velocidad de filtración glomerular. 'Índice de masa corporal; ${ }^{\ddagger}$ Velocidad de filtrado glomerular.

Tabla 2. Gravedad de la enfermedad coronaria según menopausia y diabetes mellitus tipo 2

\begin{tabular}{|c|c|c|c|c|c|c|c|c|}
\hline & \multirow[b]{2}{*}{ Total } & \multicolumn{3}{|c|}{ Mujeres premenopáusica } & \multicolumn{3}{|c|}{ Mujeres postmenopáusica } & \multirow[b]{2}{*}{ Valor $\mathbf{p}^{*}$} \\
\hline & & No DM2 & DM2 & Total & No DM2 & DM2 & Total & \\
\hline \multicolumn{9}{|l|}{$\mathrm{N}^{\circ}$ vasos } \\
\hline 0 & $241(34,1)$ & $42(47,7)$ & $14(35,0)$ & $56(43,8)$ & $134(39,5)$ & $51(21,3)$ & $185(32,0)$ & 0,01 \\
\hline 1 & $172(24,3)$ & $26(29,5)$ & $6(15,0)$ & $32(25,0)$ & $84(24,8)$ & $56(23,3)$ & $140(24,2)$ & \\
\hline 2 & $142(20,1)$ & $16(18,2)$ & $10(25,0)$ & $26(20,3)$ & $63(18,6)$ & $53(22,1)$ & $116(20,0)$ & \\
\hline 3 & $152(21,5)$ & $4 \quad(4,5)$ & $10(25,0)$ & $14(10,9)$ & $58(17,1)$ & $80(33,3)$ & $138(23,8)$ & \\
\hline \multicolumn{9}{|l|}{$\mathrm{OCT}^{+}$} \\
\hline No & $592(83,9)$ & $87(98,9)$ & $29(72,5)$ & $116(90,6)$ & $289(85,3)$ & $187(72,8)$ & $476(82,4)$ & 0,01 \\
\hline Sí & $114(16,1)$ & $1 \quad(1,1)$ & $11(27,5)$ & $12(9,4)$ & $50(14,7)$ & $52(21,8)$ & $102(17,6)$ & \\
\hline
\end{tabular}

${ }^{*} \mathrm{p} \leq 0,05$. ${ }^{\dagger}$ Oclusión crónica total. 
Tabla 3. Modelo de regresión logística simple y múltiple para presencia de enfermedad de múltiples vasos

\begin{tabular}{|c|c|c|c|c|}
\hline \multirow[b]{2}{*}{ Variables } & \multirow{2}{*}{$\begin{array}{c}\text { Modelo } 0 \\
\text { Regresiones logísticas simples } \\
\text { OR (IC 95\%) }\end{array}$} & \multicolumn{3}{|c|}{$\begin{array}{l}\text { Modelo } 1 \\
\text { Regresión logística múltiple }\end{array}$} \\
\hline & & Valor p* & OR (IC 95\%) & Valor $\mathbf{p}^{*}$ \\
\hline $\begin{array}{l}\text { Menopausia } \\
\text { Sí }\end{array}$ & $1,719(1,143 ; 2,586)$ & 0,006 & $1,787(1,079 ; 2,958)$ & 0,024 \\
\hline $\mathrm{HTA}^{\dagger}$ & & & & \\
\hline Sí & $1,662(1,177 ; 2,347)$ & 0,004 & $1,092(0,706 ; 1,689)$ & 0,694 \\
\hline $\mathrm{DM} 2^{*}$ & & & & \\
\hline Sí & $2,444(1,792 ; 3,331)$ & $<0,001$ & $1,974(1,377 ; 2,829)$ & $<0,001$ \\
\hline Tabaco & & & & \\
\hline Sí & $1,344(0,894 ; 2,019)$ & 0,155 & $1,585(0,983 ; 2,556)$ & 0,059 \\
\hline Falla renal & & & & \\
\hline Sí & $1,677(1,186 ; 2,372)$ & 0,003 & $1,499(1,041 ; 2,157)$ & 0,029 \\
\hline
\end{tabular}

${ }^{*} p \leq 0,05$. ${ }^{\dagger}$ Hipertensión arterial, ${ }^{*}$ Diabetes mellitus tipo 2 .

riables hipertensión, DM2 y compromiso renal estuvieron asociadas a mayor compromiso de múltiples vasos (Tabla 3 ).

\section{Discusión}

Se ha observado un incremento en la incidencia de enfermedad cardiovascular después de la menopausia, sin embargo, no todos los estudios están de acuerdo con este hallazgo ${ }^{10,11}$. Sabemos que el inicio de la enfermedad coronaria en la mujer es más tardío que en el hombre y mujeres jóvenes sufren menos enfermedad coronaria debido al efecto protector de los estrógenos, lo cual ayuda a prevenir la aterosclerosis ${ }^{3}$.

$\mathrm{Al}$ momento de realizar este estudio no encontramos publicaciones en nuestro país respecto de la gravedad de la enfermedad coronaria en mujeres pre y postmenopáusicas y menos aun el impacto de la DM2 en estos grupos etarios del género femenino.

Este estudio consideró como punto de corte la edad de 55 años para separar a las mujeres pre y postmenopáusicas, aun cuando la mayoría de los estudios utilizan la edad de 50 años. Consideramos esta edad como punto de corte para asegurar que prácticamente todas las pacientes catalogadas como postmenopáusicas pertenecían a este grupo. El corte de edad a los 50 años habría incrementado la población de las postmenopáusicas, pero habría debilitado la aseveración de que todas ellas eran efectivamente postmenopáusicas. La edad promedio de menopausia, en la mayoría de los países que llevan registros de salud, es de 48 años. En Venezuela es de 48,7 años \pm 4,6 años al igual que en Cuba y en la mayoría de los países de América Latina. En España, según estudios, es a partir de los 50 años ${ }^{15,16}$.

En este estudio se observó una menor prevalencia de HA y DM2 en las mujeres premenopáusicas, factores que habitualmente se van incrementando en frecuencia con la edad de las personas. Similares resultados están descritos por Lu Yihua ${ }^{8}$. El hallazgo en este estudio de un mayor compromiso de la función renal en postmenopáusicas, probablemente, esté relacionado con la alta prevalencia de estos dos factores de riesgo considerados ambos con potencial para daño renal. Existe concordancia entre el aumento en la incidencia de enfermedad coronaria en mujeres postmenopáusicas y el aumento de la prevalencia de factores de riesgo como DM2 e HA en este grupo etario. Se sabe que la DM2 genera un mayor riesgo de padecer enfermedad cardiovascular tanto en la pre como en la postmenopausia ${ }^{12}$, atenuando incluso el efecto protector de los estrógenos en la mujer en la premenopausia ${ }^{12,13}$. Los resultados observados en este estudio corroboran lo publicado, cuando se observó un significativo mayor compromiso de 
múltiples vasos y mayor frecuencia de oclusión crónica en las mujeres con DM2, tanto pre como postmenopáusicas, corroborando lo descrito en la literatura acerca de las consecuencias negativas de este factor de riesgo en los pacientes con DM2. Otra interesante observación fue que hubo más enfermedad coronaria en la paciente premenopáusica con DM2 que en la postmenopáusica sin DM2 ( $25 \%$ vs $17,1 \%)$.

P. Mellen ${ }^{14}$, en su publicación del año 2006, investiga especificamente el rol del síndrome metabólico y el de la diabetes mellitus en el mayor riesgo de enfermedad coronaria de las mujeres postmenopáusicas, demostrando que es la diabetes mellitus y no el síndrome metabólico el predictor del progreso de la enfermedad coronaria en mujeres.

En general, los resultados demostraron que en ausencia de DM2, las mujeres postmenopáusicas tienen enfermedad coronaria más grave que las premenopáusicas dado el mayor compromiso de múltiples vasos en la coronariografía $(17,1 \%$ vs $4,5 \%)(\mathrm{p}<0,001)$, lo cual también ha sido reportado previamente por otros autores como Yihua y Saltiki ${ }^{8,9}$. En presencia de DM2 esto fue más marcado aun $(33 \%$ vs $17,1 \%)(\mathrm{p}<0,001)$, incluyendo, además, una significativa mayor frecuencia de oclusión crónica en los grupos con DM2, sean estas pre o postmenopáusicas $(\mathrm{p}<0,001)$. La asociación de DM2 con enfermedad coronaria más grave fue independiente de otros factores de riesgo como HA.

Al considerar la totalidad de las pacientes premenopáusicas estas tienen una mayor proporción de mujeres sin enfermedad coronaria en la angiografía $(43,8 \%)$ vs lo observado en las postmenopaúsicas $(32 \%)$ ( $\mathrm{p}<0,01)$, lo cual se observa especialmente en las no diabéticas, en que casi la mitad de ellas está sin enfermedad (47,7\%).

En la medida que la esperanza de vida aumenta, cada vez existirán más mujeres postmenopaúsicas y muchas de ellas serán diabéticas por la prevalencia en ascenso de esta enfermedad. Este estudio entonces pone una luz de alerta sobre el impacto de la DM2 en la enfermedad cardiovascular en este grupo de mujeres.

En conclusión, las mujeres postmenopáusicas tienen mayor gravedad de EC que las premenopáusicas y la DM2 en ambos grupos de mujeres se asocia a mayor gravedad de enfermedad coronaria.

\section{Limitaciones del estudio}

En este estudio hemos considerado como punto de corte la edad de 55 años, considerando a aquellas mujeres de 55 o más años como postmenopáusicas. Esta definición deriva del hecho que la edad de presentación de la menopausia no ha cambiado significativamente en las últimas décadas y en la elaboración del trabajo no se dispone de la edad exacta de la menopausia de cada paciente. Por otra parte, no registramos el tiempo de evolución de la DM2, un parámetro que puede tener importancia en la progresión de la aterosclerosis.

\section{Referencias}

1. Elveback LR, Connolly DC, Melton LJ. Coronary heart disease in residents of Rochester, Minnesota. VII. Incidence, 1950 through 1982. Mayo Clinic Proc 1986; 61 (11): 896-900.

2. Zornitzki T, Ayzenberg O, Gandelman G, Vered S, Yaskil E, Faraggi D, et al. Diabetes, but not the metabolic syndrome, predicts the severity and extent of coronary artery disease in women. QJMed 2007; 100 (11): 57581.

3. Rosano GM, Panina G. Oestrogens and the heart. Therapie 1999; 54 (3): 381-5.

4. Grundy SM. Guidelines for colesterol management: recomendations of the National Cholesterol Education Program's Adult Treatment Panel II. Heart Dis Stroke 1994; 3 (3): 123-7.

5. Isles CG, Hole DJ, Hawthorne VM, Lever AF. Relation between coronary risk and coronary mortality in women of the Renfrew and Paisley survey. comparions with man. Lancet 1992; 339 (8795): 702-6.

6. Saltiki K, Alevizaki M. Coroanry Heart disease in postmenopausal women; the role of endogenous estrogens and their receptors. Hormones (Athens) 2007; 6 (1): 9-24.

7. Gurevitz O, Jonas M, Boyko V, Rabinowitz B, Reicher-Reiss $\mathrm{H}$. Clinical profile and long-term prognosis of women menor o igual 50 years of age referred for coronary angiography for evaluation of chest pain. Am J Cardiol 2000; 85 (7): 806-9.

8. Yihua L, Yun J, Dongshen Z. Coronary artery disease in premenopausal and postmenopausal women risk factors, cardiovascular features, and recurrence. Int Heart J 2017; 58 (2): 174-9.

9. Saltiki K, Cimponeriu A, Lili K, Peppa M, Anastasiou E, Alevaski M. Severity of coronary artery disease in 
postmenopausal diabetic women. Hormones 2008; 7 (2): 148-55

10. Colditz GA, Willett WC, Stampfer MJ, Rosner B, Speizer $\mathrm{FE}$, Hennekens $\mathrm{CH}$. Menopause and the risk of coronary heart disease in women. N Engl J Med 1987; 316 (18): 1105-10.

11. Sowers JR. Diabetes mellitus and cardiovascular disease in women. Arch Intern Med 1998; 158 (6): 617-21.

12. Kaseta JR, Skafar DF, Ram JL, Jacober SJ, Sowers JR. Cardiovascular disease in the diabetic woman. J Clin Endocrinol Metab 1999; 84 (6): 1835-8.

13. Mellen PB, Cefalu WT, Herringtong DM. Diabetes, the metabolic syndrome, and angiographic progression of coronary arterial disease in postmenopausal women. Arterioscler Thromb Vasc Biol 2006; 26 (1): 189-93.

14. Lugones M. El climaterio y el síndrome del nido vacío en el contexto sociocultural. Rev Cubana Med Gen Integr 2001; 17 2): 208-8.

15. Centro Latinoamericano y Caribeño de Demografía (CELADE). América Latina y el Caribe: el envejecimiento de la población 1950-2050. Santiago de Chile: CELADE; 2003.

16. Melidonis A, Dimopoulos V, Lempidakis E, Hatzissavas J, Kouvaras G, Stefanidis A, et al. Angiographic study of coronary artery disease in diabetic patients in comparison with nondiabetic patients. Angiology 1999; 50 (12): 997-1006. 